\title{
Maternal Serum VEGF Predicts Abnormally Invasive Placenta Better than NT-proBNP: a Multicenter Case-Control Study
}

\author{
Alexander Schwickert ${ }^{1}$ (D) . Frédéric Chantraine ${ }^{2} \cdot$ Loreen Ehrlich $^{1} \cdot$ Wolfgang Henrich $^{1} \cdot$ Mustafa Zelal Muallem $^{3}$. \\ Andreas Nonnenmacher ${ }^{1} \cdot$ Philippe Petit $^{2} \cdot$ Katharina Weizsäcker $^{1} \cdot$ Thorsten Braun $^{1,4}$
}

Received: 4 May 2020 / Accepted: 14 September 2020 / Published online: 6 October 2020

(C) The Author(s) 2020

\begin{abstract}
The aim of this study was to test if maternal serum vascular endothelial growth factor (VEGF) or N-terminal pro B-type natriuretic peptide (NT-proBNP) predicts abnormally invasive placenta (AIP) better. Secondary objective was to test whether the serum levels of VEGF and NT-proBNP can predict the degree of invasion. In a multicenter case-control study design, gestational age-matched serum samples from pregnant women with AIP $(n=44)$ and uncomplicated pregnancies $(n=55)$ who had been enrolled at Charité - Universitätsmedizin Berlin, Germany and Centre Hospitalier Régional de la Citadelle in Liège, Belgium were analyzed. Maternal blood serum VEGF and NT-proBNP levels were immunoassayed from samples taken immediately before delivery (GA median: 35 weeks). Biomarker levels were compared between AIP and control group. The correlation of biomarker levels with the clinical AIP degree was assessed. The predictive biomarker ability was characterized through a multivariate regression model and receiver operating characteristic curves. Women with AIP had significantly lower maternal serum VEGF levels (AIP mean 285 pg/ml, 95\% CI 248-322, vs. control: 391 pg $/ \mathrm{ml}, 95 \%$ CI 356-426, $p<0.01$ ) and higher NTproBNP levels (AIP median $329 \mathrm{pg} / \mathrm{ml}$, IQR 287-385, vs. control $295 \mathrm{pg} / \mathrm{ml}$, IQR 273-356, $p=0.03$ ). Maternal serum VEGF levels were able to predict AIP better (AUC $=0.729,0.622-0.836, p<0.001$; VEGF + number of previous cesarean deliveries: AUC $=0.915,0.853-0.977, p<0.001)$. Maternal serum VEGF levels correlated inversely with the clinical AIP degree $(r=-$ $0.32, p<0.01)$. In short, maternal serum VEGF, more than NT-proBNP, can help in predicting AIP and hints at the degree of invasion.
\end{abstract}

Keywords Vascular endothelial growth factor $\cdot$ Placenta increta $\cdot$ Placenta percreta $\cdot$ Abnormally invasive placenta $\cdot$ Placenta accreta spectrum $\cdot$ Biomarker

Alexander Schwickert

alexander.schwickert@charite.de

1 Department of Obstetrics, Charité - Universitätsmedizin Berlin, Corporate Member of Freie Universität Berlin, Humboldt-Universität zu Berlin, and Berlin Institute of Health, Augustenburger Platz 1, 13353 Berlin, Germany

2 Department of Obstetrics and Gynecology, Centre Hospitalier Universitaire de Liège, site CHR Citadelle, Blv 12ème de Ligne 1, 4000 Liège, Belgium

3 Department of Gynecology with Center for Oncological Surgery, Charité - Universitätsmedizin Berlin, Corporate Member of Freie Universität Berlin, Humboldt-Universität zu Berlin, and Berlin Institute of Health, Augustenburger Platz 1, 13353 Berlin, Germany

4 Department of Experimental Obstetrics, Charité -

Universitätsmedizin Berlin, Corporate Member of Freie Universität Berlin, Humboldt-Universität zu Berlin, and Berlin Institute of Health, Augustenburger Platz 1, 13353 Berlin, Germany

\section{Introduction}

Abnormally invasive placenta (AIP) describes the clinical situation where a placenta does not separate spontaneously at delivery and cannot be removed without causing abnormal and potentially life-threatening bleeding $[1,2]$.

Safe delivery in pregnancies complicated by AIP depends on the timely referral of a pregnant woman to a center specialized in treating placental pathologies, especially in the case of placenta percreta (AIP degree of invasion 3A to $3 \mathrm{C}$ according to FIGO classification) [3]. Ultrasound as the gold standard for diagnosing AIP in specialized hands yields a sensitivity and specificity of over 90\% [4-7]. However, recent population studies from the UK, the USA, and northern Europe have shown that AIP remains undiagnosed before delivery in between half to two thirds of cases [8-10]. Reasons include limited availability of specialized ultrasound scans that are 
able to detect the frequently subtle ultrasound features of AIP entities such as invasion of the lower posterior bladder wall or the parametria [11-13]. Therefore, suitable maternal serum biomarkers might aid doctors additionally to ultrasound in gynecological practices worldwide in detecting AIP early and for planning the appropriate management for pregnant women with AIP [14-16].

Vascular endothelial growth factor (VEGF) and N-terminal pro B-type natriuretic peptide (NT-proBNP) are known to play a role in the process of placentation [17-20]. Both show altered serum levels in AIP cases, having potential as promising biomarker candidates $[21,22]$. However, there has been no evaluation of these candidates against the background of different degrees of invasion of AIP, and their ability to predict AIP has yet to be comprehensively compared. VEGF promotes angiogenesis, chemotaxis, and vasodilation. It is expressed by the amniotic epithelium and the cytotrophoblast $[17,18]$. In women with AIP (Wehrum et al.: $n=13$, Uyanikoglu et al.: $n=22$ ), maternal serum VEGF levels during third trimester are significantly lower compared with women with normal placentation (0.8 (0.02-3.4) vs $6.5(2.7-10.5) \mathrm{pg} / \mathrm{mL}, p=0.02 ; 39.18 \pm$ 11.98 vs $85.87 \pm 18.05 \mathrm{pg} / \mathrm{ml}, p<0.001)$ [21, 23]. However, Biberoglu et al. did not find significantly differing serum levels for VEGF in AIP cases compared with normal placentation $(n=65)$ [24]. NT-proBNP has recently been shown to enhance vasculogenesis [20]. As women with early- (365 (149815) $\mathrm{pg} / \mathrm{ml}$ ) and late-onset preeclampsia (176 (332547) $\mathrm{pg} / \mathrm{ml}$ ) have higher plasma levels compared with controls of similar gestational age $(p<0.001)$, a placental production of NT-proBNP has been hypothesized [19]. Women with AIP seem to exhibit higher serum NT-proBNP levels than women with normal placentation [22]. It therefore appears plausible that NT-proBNP plays a role in placental vasculogenesis and development.

The aim of this study was to compare maternal serum VEGF and NT-proBNP-levels in women with different degrees of AIP and a high share of women with placenta percreta and to test whether these biomarker levels can predict the clinical degree of invasion.

\section{Methods}

\section{Study Design and Patient Population}

In a case-control study design, maternal blood serum samples were taken immediately before delivery from 99 women with singleton pregnancies enrolled prospectively at Charité Universitätsklinikum in Berlin, Germany, and Centre Hospitalier Régional de la Citadelle in Liège, Belgium. The study group consisted of 44 patients ( $n=17$ in Liège, $n=27$ in Berlin; gestational age [GA] median 35 weeks; interquartile range (IQR) 35-36 weeks) who had been diagnosed with AIP antenatally. Blood specimens taken immediately before delivery from 55 healthy women pregnant with singletons, who had been previously recruited at the Charité Universitätsklinikum during another trial, were matched for GA (median 35 weeks; IQR: 33-37 weeks) retrospectively serving as controls [25-27]. Women with conditions that might influence serum VEGF and NT-proBNP levels such as cancer, preeclampsia, hypertension, or heart failure were not included in the study. All women had provided signed informed consent for blood retrieval and serum analyses under protocols approved by the Ethics Committees of Charité Universitätsklinikum Berlin (EA1_031_15, EA2_149_07) and Centre Hospitalier Régional de la Citadelle in Liège (B412201319082).

\section{Timing of Delivery}

Planning of the delivery was done according to FIGO consensus guidelines and the evidence-based guidelines for the management of AIP which recommend to "continue expectant management until after $36+0$ weeks' gestation for women with no previous history of preterm delivery $(<36+0$ weeks) and who are stable with no vaginal bleeding, preterm premature rupture of membranes, or uterine contractions suggestive of pre-term labor" [2, 28].

\section{Ultrasonographic Evaluation and Diagnosis of Abnormally Invasive Placenta}

The prenatal diagnosis of AIP was made through grayscale, pulsed wave and color Doppler ultrasound imaging based on the previously published standardized ultrasound descriptors of AIP [29]. Most women were referred after suspicion of AIP during second trimester ultrasound scans. Women who did not have a detailed second trimester ultrasound scan were diagnosed upon their first visit to the clinic for counseling about birth mode after previous cesarean section or upon presentation in the emergency room. Grading of AIP was done intraoperatively according to the classification adopted by the FIGO from grades 1 to 3C [3]. In short, grade 1 signifies placenta accreta, grade 2 signifies placenta increta, grade $3 \mathrm{~A}$ signifies placenta percreta limited to the uterine serosa, grade $3 \mathrm{~B}$ signifies placenta percreta with urinary bladder invasion, and grade $3 \mathrm{C}$ signifies placenta percreta with invasion of other pelvic tissue/organs [3].

\section{Blood Collection, Storage, and Immunoassay Procedures}

All maternal blood samples were retrieved by venipuncture. To avoid surgery- or birth-associated affection of the biomarker levels, blood samples were taken 
preoperatively in cases of planned cesarean deliveries or at admission to the hospital in cases of attempted vaginal birth. Serum samples were spun at $3.500 \times g$ at room temperature for $10 \mathrm{~min}$; the supernatant was aliquoted and immediately stored at $-80{ }^{\circ} \mathrm{C}$ until VEGF and NTproBNP levels were measured using specific immunoassays [24]. Enzyme-linked immunosorbent assays for human unbound VEGF-A and NT-proBNP were performed according to the manufacturer's instructions (VEGF-A: Product No. SEA143Hu, Cloud-Clone Corp., Katy, USA; NT-proBNP: Catalog No. E01B0203, BlueGene, Shanghai, China) with an immunoassay device (FLUOstar Omega, BMG Labtech, Ortenberg, Germany) by investigators blinded to the patients' group classification. Both assays were run with positive controls and validated with the inter-assay coefficient of variability (VEGF: 4.5\%, NT-proBNP: $8.5 \%$ ).

\section{Statistical Analysis}

Data were tested for normality assessing the histogram and using the Kolmogorov-Smirnov test. Comparisons between groups were performed using Student's $t$ test for independent samples and Mann-Whitney $U$ test. Categorical variables were expressed as numbers (percentage) and compared using chi-square test. Data are reported as median and interquartile range (IQR) or mean and $95 \%$ confidence interval $(95 \% \mathrm{CI})$, as appropriate. Sample size calculation for the primary outcome of AIP vs. normal placentation was based on the publications by Uyanikoglu and Ersoy $(\alpha=0.05 \%$, power $=90 \%, t$ test/ANOVA) [22, 23]. It was estimated that at least 6 patients per group would be necessary to detect differences between five groups and at least 31 per group for two groups. Correlations between variables were explored using Spearman's rank order correlations for non-normally distributed continuous variables. A multivariate regression model was used to indicate predictors of AIP. In a first step, crude odds ratios with $95 \%$ confidence interval $(95 \% \mathrm{CI})$ were calculated for each variable separately (univariate analysis). In a second step, adjusted odds ratios were calculated with multivariate regression, including variables that had been identified as having a significant effect in univariate analysis (i.e., $p<0.05$ ). The true-positive and false-positive rates for the diagnosis of AIP were estimated from receiveroperating characteristic (ROC) curves, and the diagnostic powers of the biomarker levels were assessed from the respective area under the curve (AUC). Cut-off values for maternal serum VEGF and NT-proBNP levels were calculated using Youden's index [30, 31]. SPSS Version 25 (IBM, Chicago, USA) was used for statistical analysis. Figures were prepared with Prism 8 (GraphPad Software, San Diego, USA). In all analyses, two-tailed $p \leq 0.05$ were considered to indicate statistical significance.

\section{Results}

\section{Clinical Characteristics of Women and Management of AIP}

Demographic, clinical, and pregnancy outcome characteristics of the cohort are presented in Table 1. As expected, women with AIP were significantly older (34 (26-38) vs. $30(32-37)$ years, $p<0.001)$, of higher gravidity (4 (3-5) vs. $2(2-4), p<0.001)$ and parity $(2(1-3)$ vs. $1(1-2), p<0.001)$ and had a higher number of prior cesarean deliveries when compared with controls $(2(1-3)$ vs. $0(0), p<0.001)$ (Table 1). Placenta previa was diagnosed in $86 \%$ of women with AIP and in $9 \%$ of pregnancies with normal placentation. Mean gestational age at diagnosis in the two study centers based on ultrasound findings was 27 weeks (95\% CI 25-29 weeks). There was no significant difference between the two centers in Belgium and Germany (28 (26-31) vs. 26 (24-29) weeks at diagnosis, $p=0.34$ ). In an intensive preoperative counseling, the management of AIP was discussed with the patient and primarily based on the wish and in consent with the patient. Four women preferred a conservative approach with leaving the placenta in situ. Three of them experienced successful placental resorption without further procedures. One had to undergo delayed hysterectomy 3 months after cesarean section. Focal resection was performed in women who wished to conserve their uterus provided that sufficient myometrium for an optimum closure of the uterus was available after the resection. Overall, $73 \%$ of women with AIP were clinically classified as AIP degree $3 \mathrm{~A}$ to $3 \mathrm{C}$ (i.e., placenta percreta) and histopathologically confirmed. In the AIP group, 30 women (68\%) received a hysterectomy (3 (43\%) in AIP Grade 2, $3(75 \%)$ in AIP Grade 3, $17(74 \%)$ in AIP Grade 4, $5(63 \%)$ in AIP Grade 5, $2(100 \%)$ in AIP Grade 6). Statistically significant $p$-values $(<0.05)$ are written in italics.

\section{Circulating Levels of VEGF and NT-proBNP and the Occurrence of Abnormally Invasive Placenta}

VEGF levels in the AIP group were significantly lower than in the control group (AIP mean $285 \mathrm{pg} / \mathrm{ml}, 95 \%$ CI 248-322 vs. control $391 \mathrm{pg} / \mathrm{ml}, 95 \%$ CI 356-426, $p<0.01$ ) (Fig. 1a). NTproBNP levels were significantly higher in the AIP group versus the control group (AIP median $329 \mathrm{pg} / \mathrm{ml}$, IQR 287385 vs. control 295 pg/ml, IQR 273-356, $p=0.03$ ) (Fig. 1b).

\section{Correlation of Maternal Serum VEGF Level and AIP Degree}

Maternal serum VEGF levels exhibited a significantly negative correlation with the FIGO AIP degree of invasion (Spearman's rho $=-0.37 ; p<0.001$ ). This correlation remained significant even after controlling for the influence of gestational age at blood sampling through semi-partial 
Table 1 Demographic, clinical, and outcome characteristics of enrolled women $(n=99)$

\begin{tabular}{|c|c|c|c|}
\hline Variable & AIP (Grades $1-3 \mathrm{C})(n=44)$ & Controls $(n=55)$ & $P$ value \\
\hline \multicolumn{4}{|l|}{ Characteristics at enrolment } \\
\hline Maternal age (year) ${ }^{\mathrm{a}}$ & $34(26-38)$ & $30(32-37)$ & $<0.001$ \\
\hline $\mathrm{BMI}^{\mathrm{a}}$ & $29(27-30)$ & $28(26-29)$ & 0.29 \\
\hline Gravidity $^{\mathrm{b}}$ & $4(3-5)$ & $2(2-4)$ & $<0.001$ \\
\hline Parity ${ }^{\mathrm{b}}$ & $1(1-2)$ & $2(1-3)$ & $<0.001$ \\
\hline \multicolumn{3}{|l|}{ Number of prior $\mathrm{D} \& \mathrm{C}^{\mathrm{c}}$} & 0.711 \\
\hline 0 (no prior $\mathrm{D} \& \mathrm{C}$ ) & $27(61)$ & $34(62)$ & \\
\hline 1 prior $\mathrm{D} \& \mathrm{C}$ & $11(25)$ & $11(20)$ & \\
\hline$\geq 2$ prior $\mathrm{D} \& \mathrm{C}$ & $6(14)$ & $10(18)$ & \\
\hline \multicolumn{3}{|l|}{ Number of prior cesarean deliveries ${ }^{\mathrm{c}}$} & $<0.001$ \\
\hline 0 (no prior cesareans) & $4(9)$ & $43(78)$ & \\
\hline 1 prior cesarean & $17(39)$ & $9(16)$ & \\
\hline$\geq 2$ prior cesareans & $23(52)$ & $3(6)$ & \\
\hline Placenta previa ${ }^{\mathrm{c}}$ & $38(86)$ & $5(9)$ & $<0.001$ \\
\hline \multicolumn{4}{|l|}{ Outcome characteristics } \\
\hline Gestational age at delivery in weeks ${ }^{\mathrm{b}}$ & $35(35-36)$ & $35(33-37)$ & 0.397 \\
\hline Cesarean delivery $^{\mathrm{c}}$ & $44(100)$ & $18(33)$ & $<0.001$ \\
\hline Cesarean hysterectomy ${ }^{\mathrm{c}}$ & $30(68)$ & 0 & $<0.001$ \\
\hline Focal resection ${ }^{\mathrm{c}}$ & $7(16)$ & 0 & $<0.001$ \\
\hline Leaving placenta in $s^{i t u}{ }^{c}$ & $4(9)$ & 0 & $<0.001$ \\
\hline Manual removal of placenta ${ }^{c}$ & $4(9)$ & 0 & $<0.001$ \\
\hline \multicolumn{3}{|l|}{ AIP grading ${ }^{\mathrm{c}}$} & $<0.001$ \\
\hline Normal placentation & 0 & $55(100)$ & \\
\hline 1 & $6(13.6)$ & 0 & \\
\hline 2 & $6(13.6)$ & 0 & \\
\hline $3 \mathrm{~A}$ & $22(50)$ & 0 & \\
\hline $3 \mathrm{~B}$ & $7(16)$ & 0 & \\
\hline $3 \mathrm{C}$ & $3(6.8)$ & 0 & \\
\hline Blood loss in milliliter $^{\mathrm{a}}$ & $1865(1464-2266)$ & $367(305-430)$ & $<0.001$ \\
\hline \multicolumn{3}{|l|}{ Blood transfusion $^{\mathrm{c}}$} & $<0.001$ \\
\hline 0 red packed cells & $22(51)$ & $55(100)$ & \\
\hline$\leq 4$ red packed cells & $15(35)$ & 0 & \\
\hline$\geq 5$ red packed cells & $6(14)$ & 0 & \\
\hline
\end{tabular}

Abbreviations: AIP abnormally invasive placenta, $D \& C$ dilation and curettage

${ }^{\text {a }}$ Data presented as mean (95\% CI) and analyzed by $t$ test

${ }^{\mathrm{b}}$ Data presented as median (IQR) and analyzed by Mann-Whitney $U$ test

${ }^{\mathrm{c}}$ Data presented as $n(\%)$ and analyzed by chi-squared test correlation $(r=-0.33, p<0.01)$. Figure 1c illustrates this correlation but shows that no linear negative correlation from normal placentation to AIP degrees of invasion $3 \mathrm{~B}+3 \mathrm{C}$ exists, as the median of VEGF in AIP degree of invasion 2 (placenta increta) and 3A (placenta percreta) is higher than for AIP grade 1 (placenta accreta). Grouping of grades 3B and $3 \mathrm{C}$ was performed due to limited case numbers in the subgroups and based on the assumption that these AIP degrees both denote highly invasive placenta percreta, which has invaded parauterine structures such as the urinary bladder or the parametria. Maternal serum NT-proBNP levels did not correlate significantly with the AIP degree (Spearman's rho = $0.17 ; p=0.12$; Fig. 1d).

\section{Logistic Regression Analysis of Possible Risk Factors for AIP}

The predictive power of biomarker levels and the number of previous cesarean deliveries concerning the occurrence of AIP were analyzed by univariate logistic regression analysis (Table 2). Having at least one previous cesarean delivery and levels of VEGF $\leq 328.0 \mathrm{pg} / \mathrm{ml}$ or NT-proBNP $\geq$ 


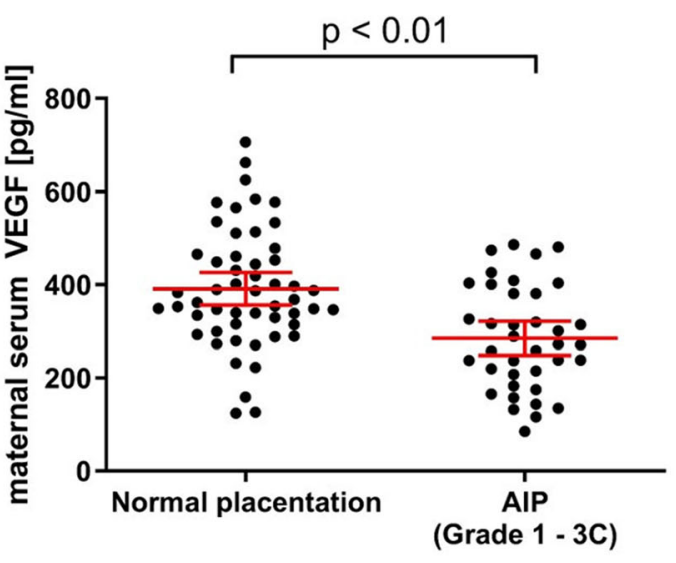

a

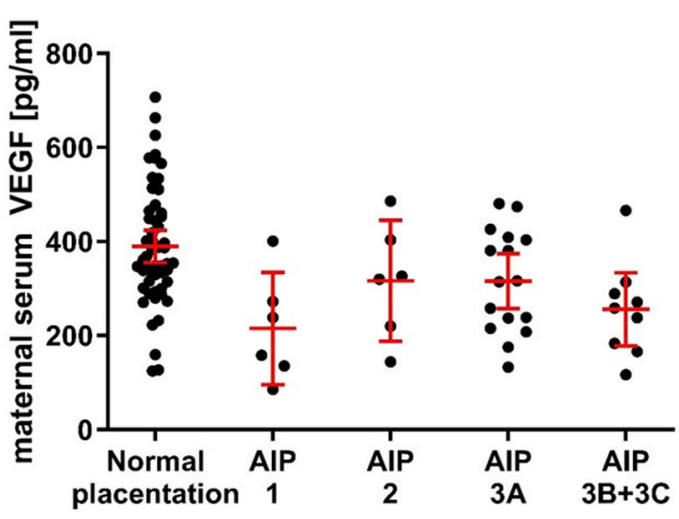

Fig. 1 Angiogenic factors in women with abnormally invasive placenta. Scatter plot showing the significant a decrease of maternal serum VEGF and $\mathbf{b}$ increase of maternal serum NT-proBNP in women with abnormally invasive placenta (VEGF: $n=38$, NT-proBNP: $n=43$ ) as compared with gestational age-matched controls $(n=54)$. Values are shown as mean \pm 95\% confidence interval $(95 \% \mathrm{CI}$ ) for VEGF and median \pm interquartile range (IQR) for NT-proBNP. The median of gestational age at delivery was 35 weeks in both groups (AIP: IQR 35-36; Controls: IQR 33-37). c,

$303.5 \mathrm{pg} / \mathrm{ml}$ were detected to be significant risk factors for the occurrence of AIP. Multivariate logistic regression analysis confirmed the determining values for VEGF, NT-proBNP, and the number of previous cesarean deliveries (Table 2). Gravidity, parity, age, and the presence of placenta previa showed a significant correlation with the number of previous cesarean sections. These factors were therefore left out of the regression analysis to avoid multicollinearity.

Table 2 Univariate and multivariate regression analyses showing the value of variables (number of prior cesarean deliveries, maternal serum VEGF, and NT-proBNP levels) to predict AIP. Due to highly significant correlation with the number of previous cesarean sections, maternal age,

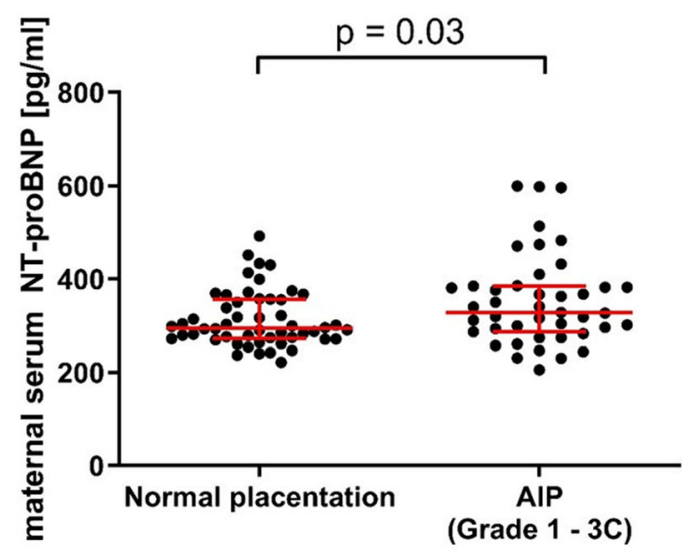

b

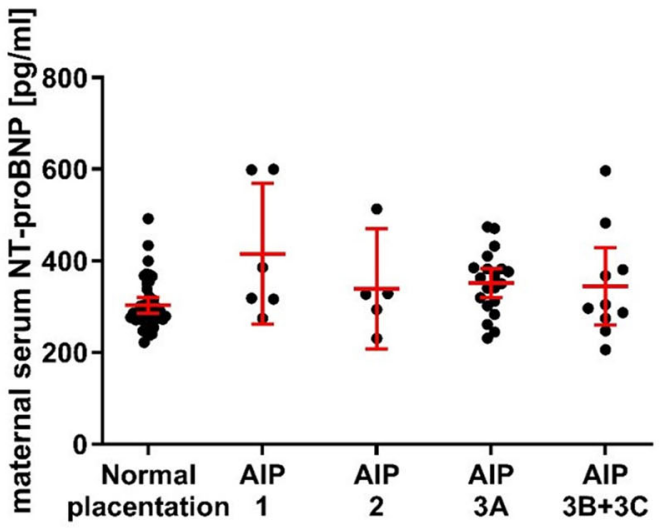

d VEGF and NT-proBNP levels according to AIP degree. Scatter plot showing maternal serum VEGF and NT-proBNP levels (mean $\pm 95 \% \mathrm{CI}$ ) in relation to AIP degree. Following clinical relevance, degrees of invasion $3 \mathrm{~B}$ and $3 \mathrm{C}$ have been clustered together based on the assumption that these AIP degrees both denote highly invasive placenta percreta, which has invaded parauterine structures such as the urinary bladder (3B) or the parametria (3C)

\section{Predictive Value of VEGF on the Occurrence and the Outcome of AIP}

The diagnostic values of maternal serum VEGF and NTproBNP levels in patients with AIP are summarized in Fig. 2 and Table 3. NT-proBNP level with a cut-off at $303.5 \mathrm{pg} / \mathrm{ml}$ had a negative likelihood ratio (LR-) of 0.6 and a positive likelihood ratio $(\mathrm{LR}+)$ of 1.6 for the outcome of AIP

gravidity, and parity are left out in regression analysis to avoid distortion due to multicollinearity. Statistically significant $p$-values $(<0.05)$ are written in italics

\begin{tabular}{|c|c|c|c|c|}
\hline & Crude OR $(95 \% \mathrm{CI})$ & $P$ value & Adjusted OR (95\% CI) & $P$ value \\
\hline Number of prior cesarean deliveries & $7.5(3.4-16.8)$ & $<0.001$ & $5.7(2.5-13.1)$ & $<0.001$ \\
\hline VEGF $\leq 328.0 \mathrm{pg} / \mathrm{ml}$ & $6.3(2.5-15.6)$ & $<0.001$ & $3.4(1.1-11.0)$ & 0.041 \\
\hline NT-proBNP $\geq 303.5 \mathrm{pg} / \mathrm{ml}$ & $2.9(1.3-6.6)$ & 0.01 & $3.7(1.1-12.5)$ & 0.038 \\
\hline
\end{tabular}

Abbreviations: VEGF vascular endothelial growth factor, NT-proBNP N-terminal pro B-type natriuretic peptide, OR (95\% CI) odds ratio (95\% confidence interval) 


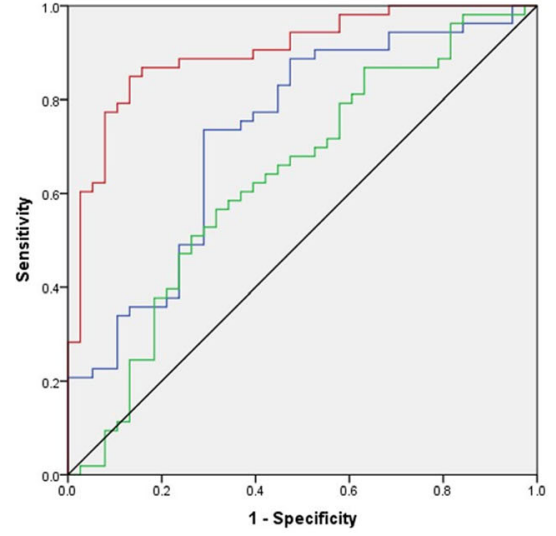

a) outcome: AIP

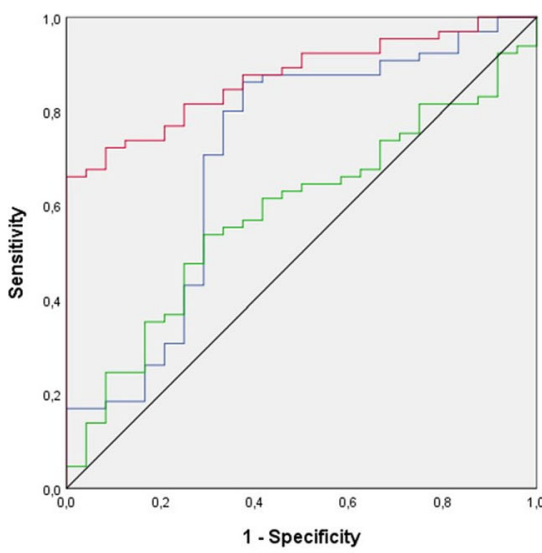

b) outcome: peripartum hysterectomy
- serum NT-proBNP

— serum VEGF

- serum VEGF and

number of previous

cesarean sections

Reference line
Fig. 2 ROC curves showing the predictive value of serum VEGF and NT-proBNP in AIP. a Predictive value of serum biomarkers alone and in a combination of serum VEGF with the number of previous cesarean sections on the occurrence of AIP (outcome: Grading 2 to 6, VEGF: $\mathrm{AUC}=0.729$; NT-proBNP: $\mathrm{AUC}=0.632$; serum VEGF and previous cesarean deliveries: $A U C=0.915)$. b Predictive value of serum

(AUC $=0.632,95 \%$ CI $0.516-0.747, p=0.03)$. A VEGF level cut-off at $328.0 \mathrm{pg} / \mathrm{ml}$ had a negative likelihood ratio of 0.4 and a positive likelihood ratio of 2.5 for the same outcome (AUC $=0.729,95 \%$ CI $0.622-0.836, p<0.001)$. A combination of maternal serum VEGF level and the number of prior cesarean deliveries even increased the predictive value concerning the occurrence of AIP (AUC $=0.915,95 \% \mathrm{CI}$ 0.853-0.977, LR- 0.1, LR+ 6.7, $p<0.001)$. The predictive value of the maternal serum VEGF level combined with previous cesarean sections was significantly better than the predictive value of the number of previous cesarean sections alone (AUC difference 0.038, $p=0.04$ ). Combining VEGF and NT-proBNP levels did not yield a better predictive power than VEGF alone (AUC $=0,729,95 \%$ CI $0,620-0,832, p<$ $0.001)$. Serum VEGF was able to predict the need for peripartum hysterectomy $(\mathrm{AUC}=0.698,95 \% \mathrm{CI} 0.564-0.832$, LR+ $2.4, \mathrm{LR}-0.3, p=0.004)$. To show that this finding was not influenced by potentially different management in the two biomarkers and the need for peripartum hysterectomy (VEGF: AUC $=$ 0.698; NT-proBNP: AUC $=0.585$; serum VEGF and previous cesarean deliveries: $\mathrm{AUC}=0.870$ ). There was no significant difference in the hysterectomy rate between the two study centers in Liège and Berlin ( $p=$ 0,975 , chi-squared test)

study centers, chi-squared test was performed and showed no significant difference in the hysterectomy rate between Liège and Berlin $(p=0.975)$.

\section{Discussion}

Due to its subtle ultrasound features, AIP is difficult to detect for the untrained eye. This reflects in detection rates of 50 to $66 \%$ in population studies [8-10], despite high sensitivity and specificity of ultrasound in the hands of an expert [4]. A better detection of AIP through the use of biomarkers could lead to a more convenient planning of scheduled cesarean delivery with a prepared expert team, which will result in a better outcome for the patient with less blood loss and less hysterectomies $[8$, 32, 33]. The study has confirmed the existence of altered maternal serum levels of both investigated angiogenic biomarkers in pregnancies affected by AIP. Serum NT-proBNP

Table 3 Sensitivity, specificity, negative and positive predictive value, likelihood ratios, and area under the curve (AUC) values of determined VEGF and NT-proBNP cut-off levels in patients with AIP

$\begin{array}{llllllll}\text { Cut-off } & \text { Sensitivity } & \text { Specificity } & \text { NPV } & \text { PPV } & \text { LR } & \text { LR+ } & \text { AUC }(95 \% \mathrm{CI})\end{array}$

\begin{tabular}{|c|c|c|c|c|c|c|c|c|c|}
\hline VEGF & $328.0 \mathrm{pg} / \mathrm{ml}$ & $73.6 \%$ & $71.1 \%$ & $78.9 \%$ & $64.7 \%$ & 0.4 & 2.5 & $0.729(0.622-0.836)$ & $<0.001$ \\
\hline NT-proBNP & $303.5 \mathrm{pg} / \mathrm{ml}$ & $65.1 \%$ & $60.4 \%$ & $68.5 \%$ & $56.7 \%$ & 0.6 & 1.6 & $0.632(0.516-0.747)$ & 0.03 \\
\hline Number of previous CS & & $91.2 \%$ & $77.4 \%$ & $93.2 \%$ & $72.1 \%$ & 0.1 & 4.0 & $0.881(0.805-0.957)$ & $<0.001$ \\
\hline VEGF and number of previous CS & & $88.2 \%$ & $86.8 \%$ & $92.0 \%$ & $81.0 \%$ & 0.1 & 6.7 & $0.915(0.853-0.977)$ & $<0.001$ \\
\hline NT-proBNP and number of previous CS & & $85.3 \%$ & $83.0 \%$ & $89.8 \%$ & $76.2 \%$ & 0.2 & 5.0 & $0.904(0.843-0.965)$ & $<0.001$ \\
\hline
\end{tabular}

Statistically significant $p$ values $(<0.05)$ are written in italics

Abbreviations: $C S$ cesarean sections, $V E G F$ vascular endothelial growth factor, $N T$-proBNP N-terminal pro B-type natriuretic peptide, $A U C$ area under the curve, $N P V$ negative predictive value, $P P V$ positive predictive value, $L R-$ negative likelihood ratio, $L R+$ positive likelihood ratio 
level is significantly increased in cases of AIP, while serum VEGF level was significantly reduced compared with controls, even when controlled for the number of prior cesarean deliveries per woman - the main clinical risk factor for AIP. Combining maternal serum VEGF level and the number of previous cesarean deliveries through ROC curve analysis yielded the highest predictive value on the occurrence of AIP - significantly higher than the predictive value of the number of previous cesarean sections alone. Decreased maternal serum VEGF levels significantly predicted the higher probability and need to perform a peripartum hysterectomy. This shows that serum VEGF level can be a useful part of an algorithm screening for AIP based on combined predictive values together with clinical characteristics.

Serum VEGF levels correlated inversely but not linearly with the AIP degree of invasion after controlling for gestational age at blood retrieval. Correlation analysis between serum NT-proBNP levels and the AIP degrees of invasion showed a trend but was not significant. NT-proBNP therefore seems to be of limited ability to predict AIP. VEGF appears to be the better biomarker for AIP. The question is, which pathophysiological process of AIP leads to the observed alterations in maternal serum VEGF and NT-proBNP levels? It is known that placental invasiveness is promoted by neoangiogenesis $[34,35]$. Subplacental and uterovesical hypervascularity in AIP presents both in antenatal sonography [29] and in postpartum histological sections of placental beds [36]. Therefore, we hypothesized that NT-proBNP and VEGF as vasculogenic factors are elevated in maternal serum. Interestingly, in accordance with the study by Wehrum et al., who collected serum samples at an earlier mean gestational age of 31 (28-34) weeks prior to steroid administration and blood transfusion, serum VEGF levels appeared to be lower in our AIP cohort [21]. This finding could be explained by an underlying pathophysiological mechanism in AIP: the role of oxidative stress. Placental hypoxia - and therefore reduced oxidative stressstimulates increased placental invasiveness by enhancing the expression of VEGF [37]. According to the currently accepted hypothesis of AIP pathogenesis, AIP mainly develops when nidation has taken place in the region of a uterine scar - a region of localized hypoxia due to abnormal vascularization that has resulted from the scarring process after surgery [21, 38-40]. We presume that VEGF levels might be elevated during the first and second trimester, which would explain increased neoangiogenesis in AIP. When normoxia - or even hyperoxia-has been achieved through increased neoangiogenesis in AIP, augmented oxidative stress might lead to a downregulation of VEGF [41, 42]. As blood was sampled during the third trimester (median GA 35 weeks, IQR 35-36 weeks), this hypothesis could explain the paradoxically low serum VEGF levels in cases of AIP.

The strength of the study lies in the size of the cohort. Especially the comparatively large number of cases of placenta percreta (degrees of invasion 3A-3C: $n=32$ ) stands out against the sizes of the existing studies. Still no differentiation in VEGF levels between clinical AIP degrees (degrees of invasion $1-3 \mathrm{C}$ according to FIGO [3]) could be seen. This limitation shows that for further evaluation of reasonable VEGF thresholds, an even larger number of patients are needed. Although the studied patient collective of $n=44$ is large against the background of the rarity of AIP, the number of patients in the subgroups (AIP degrees of invasion 1-3C) is still rather small. For example, only three patients with AIP degree $3 \mathrm{C}$ (placental infiltration of the parametrium) were recruited in the study. Therefore, the statistical power of the subgroup analysis is limited.

Various physiological situations can alter human biomarker levels. Serum NT-proBNP levels can be increased by physiological stress and cardiac strain [43-45]. We controlled for this confounding factors by retrieving blood pre-operatively. VEGF can be altered by a person's body mass index (BMI). The individuals in the AIP group did not have significantly different BMIs compared with the control group (Table 1). A slight increase in serum VEGF levels during the course of pregnancy has been shown in other studies [46-48]. To control for this effect (which is much stronger in preeclamptic pregnancies), the groups were matched for gestational age at blood retrieval. Placenta previa does not seem to influence maternal serum levels of VEGF and NT-proBNP [21, 22, 24].

We hypothesized that biomarker levels might be altered most significantly later in pregnancy, when the process of placentation is far advanced. As most of the blood samples were drawn during third trimester, the usefulness of determining these biomarkers earlier in pregnancy - which would be the prerequisite for a useful biomarker-still must be investigated. Two studies exist $(N=48 ; N=70)$, which have documented maternal serum VEGF at 3 or 5 time points during pregnancy in women with preeclampsia, gestational hypertension, or normotension $[47,48]$. Due to this limited evidence, no normal values or standard curves according to gestational age are available for VEGF and the measured biomarker levels cannot yet be expressed as multiples of the median. However, VEGF levels appear to be very stable throughout normotensive pregnancies [47, 48]. However, the study shows that the biomarker levels are still altered in third trimester. This indicates that VEGF could also aid birth clinics in determining their management for patients who are referred late in pregnancy.

The degree of biomarker levels depends on the specific immunoassay used. For VEGF, different research groups have recorded different levels in pregnancy: $0.02-11 \mathrm{pg} / \mathrm{ml}$ (R\&D Systems, Minneapolis, USA) [21], 3-9 pg/ml (Cat No: SEA143Hu, Lot No: L150303051 USCN life science Inc., Wuhan, Hubei, China) [24], and 35-110 pg/ml (Cat No: EEL-H2569, Elabscience, Wuhan, Hubei, China) [23]. Palm et al. could only detect VEGF (above $9 \mathrm{pg} / \mathrm{ml}$ ) in $15 \%$ of 
normal pregnant women beyond 33 weeks (Cat No: DVE00, R\&D Systems, Minneapolis, MN, USA) [46]. In this study, women had VEGF levels of $240-430 \mathrm{pg} / \mathrm{ml}$. For NT-proBNP, the most favorable cut-off level to differentiate between AIP and normal placentation as determined by ROC curve analysis in this study was $303.5 \mathrm{pg} / \mathrm{ml}$. Ersoy et al. proposed a cut-off at $125.85 \mathrm{pg} / \mathrm{ml}$ (manufacturer of assay not stated in the publication) [22]. Standard curves for NT-proBNP according to gestational age are also not available. Only Franz et al. calculated multiples of the meridian using an electrochemiluminescence immunoassay $(N=94)$ [49]. Before general clinical implementation, more studies are needed to define concrete cut-off values for reliable prediction of AIP (and its degrees of invasion). Biomarkers cannot replace a detailed ultrasound scan. However, in comparison with the aforementioned AIP detection rates of 50 to $66 \%$, a routine assessment of the number of previous cesarean sections and VEGF level could lead to a relative risk reduction of 60 to $70 \%$ for the event of an unsuspected AIP [8-10].

\section{Conclusion}

Third trimester levels of VEGF, more than NT-proBNP, can help in predicting AIP and the need for hysterectomy prior to delivery - especially in combination with clinical factors such as the number of prior cesarean deliveries. Biomarker levels did not allow a clear antenatal classification of the degree of placental invasion. A maternal serum biomarker hinting at AIP can be helpful in outpatient settings where no specialized ultrasound scan is available to help to identify patients at high risk of AIP and refer them to a specialized center for further diagnostics. As a next step, we propose to determine maternal serum VEGF levels in pregnancies complicated by AIP starting from first trimester onwards.

Acknowledgments Open Access funding enabled and organized by Projekt DEAL. We thank K. Melchior and T. Ziska (Department of 'Experimental Obstetrics', Berlin, Germany) for their excellent technical assistance. We thank Dr. R.C. Rancourt for language editing and proofreading. We are also indebted to all the staff of our Departments of Obstetrics and Gynecology.

Authors' Contributions All authors contributed substantially in conception and planning of the study. AS collected the data and drafted the first manuscript. FC, AN, KW, MZM, PP, WH, and TB performed the cesarean sections. AS and LE performed the enzyme-linked immunosorbent assays. All authors contributed to the data analysis and interpretation of the data. AS and TB revised the manuscript together. All authors reviewed and approved the final submitted version of the manuscript.

Funding University research fund of Charité - Universitätsmedizin Berlin, Germany (51517172-01).

Data Availability All raw data are available upon request.

\section{Compliance with Ethical Standards}

Conflict of Interest The authors declare that they have no conflict of interest.

Ethical Approval The study was approved by the Regional Ethics Committee in Berlin, Germany, No. EA2_149_07, 02 July 2010 and No. EA1 031 15, 31 July 2015 and by the Regional Ethics Committee in Liège, Belgium, No. B412201319082 on 31 October 2017.

Consent to Participate All subjects consented to the blood retrieval as well as collection of pseudonymized clinical information as approved by the Regional Ethics Committee in Berlin, Germany, and by the Regional Ethics Committee in Liège, Belgium.

Consent for Publication All authors consent to the publication of this paper.

Code Availability Not applicable.

Open Access This article is licensed under a Creative Commons Attribution 4.0 International License, which permits use, sharing, adaptation, distribution and reproduction in any medium or format, as long as you give appropriate credit to the original author(s) and the source, provide a link to the Creative Commons licence, and indicate if changes were made. The images or other third party material in this article are included in the article's Creative Commons licence, unless indicated otherwise in a credit line to the material. If material is not included in the article's Creative Commons licence and your intended use is not permitted by statutory regulation or exceeds the permitted use, you will need to obtain permission directly from the copyright holder. To view a copy of this licence, visit http://creativecommons.org/licenses/by/4.0/.

\section{References}

1. Chantraine F, Langhoff-Roos J. Abnormally invasive placenta AIP. Awareness and pro-active management is necessary. Acta Obstet Gynecol Scand. 2013;92(4):369-71.

2. Collins SL, Alemdar B, van Beekhuizen HJ, Bertholdt C, Braun T, Calda $\mathrm{P}$, et al. Evidence-based guidelines for the management of abnormally invasive placenta recommendations from the International Society for Abnormally Invasive Placenta. Am J Obstet Gynecol. 2019;220:511-26.

3. Jauniaux E, Ayres-de-Campos D, Langhoff-Roos J, Fox KA, Collins S. FIGO classification for the clinical diagnosis of placenta accreta spectrum disorders. Int J Gynaecol Obstet. 2019;146(1):20 4.

4. Jauniaux E, Bhide A. Prenatal ultrasound diagnosis and outcome of placenta previa accreta after cesarean delivery: a systematic review and meta-analysis. Am J Obstet Gynecol. 2017;217(1):27-36.

5. Jauniaux E, Bhide A, Kennedy A, Woodward P, Hubinont C, Collins S. FIGO consensus guidelines on placenta accreta spectrum disorders: prenatal diagnosis and screening. Int J Gynaecol Obstet. 2018;140(3):274-80.

6. Delli Pizzi A, Tavoletta A, Narciso R, Mastrodicasa D, Trebeschi S, Celentano C, et al. Prenatal planning of placenta previa: diagnostic accuracy of a novel MRI-based prediction model for placenta accreta spectrum (PAS) and clinical outcome. Abdom Radiol (NY). 2019;44(5):1873-82. 
7. Lam G, Kuller J, McMahon M. Use of magnetic resonance imaging and ultrasound in the antenatal diagnosis of placenta accreta. J Soc Gynecol Investig. 2002;9(1):37-40.

8. Fitzpatrick KE, Sellers S, Spark P, Kurinczuk JJ, Brocklehurst P, Knight M. The management and outcomes of placenta accreta, increta, and percreta in the UK: a population-based descriptive study. BJOG. 2014;121(1):62-70 discussion 70-1.

9. Bailit JL, Grobman WA, Rice MM, Reddy UM, Wapner RJ, Varner MW, et al. Morbidly adherent placenta treatments and outcomes. Obstet Gynecol. 2015;125(3):683-9.

10. Thurn L, Lindqvist PG, Jakobsson M, Colmorn LB, Klungsoyr K, Bjarnadóttir RI, et al. Abnormally invasive placenta-prevalence, risk factors and antenatal suspicion: results from a large population-based pregnancy cohort study in the Nordic countries. BJOG. 2016;123(8):1348-55.

11. Palacios-Jaraquemada JM, D'Antonio F, Buca D, Fiorillo A, Larraza P. Systematic review on near miss cases of placenta accreta spectrum disorders: correlation with invasion topography, prenatal imaging, and surgical outcome. J Matern Fetal Neonatal Med. 2019;33(19):3377-3384. https://doi.org/10.1080/14767058.2019. 1570494

12. Jauniaux E, Collins SL, Jurkovic D, Burton GJ. Accreta placentation: a systematic review of prenatal ultrasound imaging and grading of villous invasiveness. Am J Obstet Gynecol. 2016;215(6): 712-21.

13. Melcer Y, Jauniaux E, Maymon S, et al. Impact of targeted scanning protocols on perinatal outcomes in pregnancies at risk of placenta accreta spectrum or vasa previa. Am J Obstet Gynecol. 2018;218(4):443.e1-8.

14. Kawashima A, Sekizawa A, Ventura W, Koide K, Hori K, Okai T, et al. Increased levels of cell-free human placental lactogen mRNA at 28-32 gestational weeks in plasma of pregnant women with placenta previa and invasive placenta. Reprod Sci. 2014;21(2):215-20.

15. Goh W, Yamamoto SY, Thompson KS, Bryant-Greenwood GD. Relaxin, its receptor (RXFP1), and insulin-like peptide 4 expression through gestation and in placenta accreta. Reprod Sci. 2013;20(8): 968-80.

16. Gu Y, Meng J, Zuo C, Wang S, Li H, Zhao S, Huang T, Wang X, Yan J. Downregulation of MicroRNA-125a in placenta accreta spectrum disorders contributes antiapoptosis of implantation site intermediate trophoblasts by targeting MCL1. Reprod Sci. 2019;26(12):1582-1589. https://doi.org/10.1177/ 1933719119828040

17. Brouillet S, Hoffmann P, Feige J-J, Alfaidy N. EG-VEGF: a key endocrine factor in placental development. Trends Endocrinol Metab. 2012;23(10):501-8.

18. Tseng JJ, Chou MM, Hsieh YT, Wen MC, Ho ESC, Hsu SL. Differential expression of vascular endothelial growth factor, placenta growth factor and their receptors in placentae from pregnancies complicated by placenta accreta. Placenta. 2006;27(1):70-8.

19. Junus K, Wikström A-K, Larsson A, Olovsson M. Placental expression of proBNP/NT-proBNP and plasma levels of NT-proBNP in early- and late-onset preeclampsia. Am J Hypertens. 2014;27(9): 1225-30.

20. Shmilovich H, Ben-Shoshan J, Tal R, Afek A, Barshack I, MayselAuslander S, et al. B-type natriuretic peptide enhances vasculogenesis by promoting number and functional properties of early endothelial progenitor cells. Tissue Eng A. 2009;15(9):27419 .

21. Wehrum MJ, Buhimschi IA, Salafia C, et al. Accreta complicating complete placenta previa is characterized by reduced systemic levels of vascular endothelial growth factor and by epithelial-tomesenchymal transition of the invasive trophoblast. Am J Obstet Gynecol. 2011;204(5):411.e1-411.e11.
22. Ersoy AO, Oztas E, Ozler S, Ersoy E, Erkenekli K, Uygur D, et al. Can venous ProBNP levels predict placenta accreta? J Matern Fetal Neonatal Med. 2016;29(24):4020-4.

23. Uyanıkoğlu H, İncebıyık A, Turp AB, Çakmak G, Sak S, Hilali NG. Serum angiogenic and anti-angiogenic markers in pregnant women with placenta percreta. Balkan Med J. 2018;35(1):55-60.

24. Biberoglu E, Kirbas A, Daglar K, Biberoglu K, Timur H, Demirtas $\mathrm{C}$, et al. Serum angiogenic profile in abnormal placentation. $\mathrm{J}$ Matern Fetal Neonatal Med. 2016;29(19):3193-7.

25. Gütling H, Bionaz M, Sloboda DM, Ehrlich L, Braun F, Gramzow $\mathrm{AK}$, et al. The importance of selecting the right internal control gene to study the effects of antenatal glucocorticoid administration in human placenta. Placenta. 2016;44:19-22.

26. Braun T, Husar A, Challis JRG, Dudenhausen JW, Henrich W, Plagemann A, et al. Growth restricting effects of a single course of antenatal betamethasone treatment and the role of human placental lactogen. Placenta. 2013;34(5):407-15.

27. Braun F, Hardt AK, Ehrlich L, Sloboda DM, Challis JRG, Plagemann A, et al. Sex-specific and lasting effects of a single course of antenatal betamethasone treatment on human placental 11ß-HSD2. Placenta. 2018;69:9-19.

28. Allen L, Jauniaux E, Hobson S, Papillon-Smith J, Belfort MA. FIGO consensus guidelines on placenta accreta spectrum disorders: nonconservative surgical management. Int J Gynaecol Obstet. 2018;140(3):281-90.

29. Collins SL, Ashcroft A, Braun T, Calda P, Langhoff-Roos J, Morel $\mathrm{O}$, et al. Proposal for standardized ultrasound descriptors of abnormally invasive placenta (AIP). Ultrasound Obstet Gynecol. 2016;47(3):271-5.

30. Youden WJ. Index for rating diagnostic tests. Cancer. 1950;3(1): 32-5.

31. Habibzadeh F, Habibzadeh P, Yadollahie M. On determining the most appropriate test cut-off value: the case of tests with continuous results. Biochem Med (Zagreb). 2016;26(3):297-307.

32. Chandraharan E, Rao S, Belli A-M, Arulkumaran S. The triple-P procedure as a conservative surgical alternative to peripartum hysterectomy for placenta percreta. Int J Gynaecol Obstet. 2012;117(2):191-4.

33. Al-Khan A, Gupta V, Illsley NP, et al. Maternal and fetal outcomes in placenta accreta after institution of team-managed care. Reprod Sci. 2014;21(6):761-71.

34. Bartels HC, Postle JD, Downey P, Brennan DJ. Placenta accreta spectrum: a review of pathology, molecular biology, and biomarkers. Dis Markers. 2018;2018:1507674.

35. Illsley NP, DaSilva-Arnold SC, Al-Khan A, Zamudio S. Trophoblast invasion: lessons from abnormally invasive placenta (placenta accreta). Placenta. 2020. https://doi.org/10.1016/j. placenta.2020.01.004

36. Chantraine F, Blacher S, Berndt S, et al. Abnormal vascular architecture at the placental-maternal interface in placenta increta. Am J Obstet Gynecol. 2012;207(3):188.e1-9.

37. Wu F, Tian F-J, Lin Y. Oxidative stress in placenta: health and diseases. Biomed Res Int. 2015;2015:293271.

38. Kobayashi H, Kawahara N, Ogawa K, Yoshimoto C. A Relationship between endometriosis and obstetric complications. https://doi.org/10.1007/s43032-019-00118-0. Updated May 1, 2020. Accessed May 1, 2020.

39. Jauniaux E, Jurkovic D. Placenta accreta: pathogenesis of a 20th century iatrogenic uterine disease. Placenta. 2012;33(4):244-51.

40. Roeder HA, Cramer SF, Leppert PC. A look at uterine wound healing through a histopathological study of uterine scars. Reprod Sci. 2012;19(5):463-73.

41. Forsythe JA, Jiang BH, Iyer NV, Agani F, Leung SW, Koos RD, et al. Activation of vascular endothelial growth factor gene transcription by hypoxia-inducible factor 1 . Mol Cell Biol. 1996;16(9):4604-13. 
42. Wan J, Lata C, Santilli A, Green D, Roy S, Santilli S. Supplemental oxygen reverses hypoxia-induced smooth muscle cell proliferation by modulating HIF-alpha and VEGF levels in a rabbit arteriovenous fistula model. Ann Vasc Surg. 2014;28(3):725-36.

43. Hall C. Essential biochemistry and physiology of (NT-pro)BNP. Eur J Heart Fail. 2004;6(3):257-60.

44. Donnellan E, Phelan D. Biomarkers of cardiac stress and injury in athletes: what do they mean? Curr Heart Fail Rep. 2018;15(2):11622.

45. Marlinge M, Coulange M, Fitzpatrick RC, Delacroix R, Gabarre A, Lainé N, et al. Physiological stress markers during breath-hold diving and SCUBA diving. Phys Rep. 2019;7(6):e14033.

46. Palm M, Basu S, Larsson A, Wernroth L, Åkerud H, Axelsson O. A longitudinal study of plasma levels of soluble fms-like tyrosine kinase 1 (sFlt1), placental growth factor (PlGF), sFlt1: PlGF ratio and vascular endothelial growth factor (VEGF-A) in normal pregnancy. Acta Obstet Gynecol Scand. 2011;90(11):1244-51.
47. Bosio PM, Wheeler T, Anthony F, Conroy R, O'herlihy C, McKenna P. Maternal plasma vascular endothelial growth factor concentrations in normal and hypertensive pregnancies and their relationship to peripheral vascular resistance. Am J Obstet Gynecol. 2001;184(2):146-52.

48. Hunter A, Aitkenhead M, Caldwell C, McCracken G, Wilson D, McClure N. Serum levels of vascular endothelial growth factor in preeclamptic and normotensive pregnancy. Hypertension. 2000;36(6):965-9.

49. Franz MB, Andreas M, Schiessl B, et al. NT-proBNP is increased in healthy pregnancies compared to non-pregnant controls. Acta Obstet Gynecol Scand. 2009;88(2):234-7.

Publisher's Note Springer Nature remains neutral with regard to jurisdictional claims in published maps and institutional affiliations. 